Recent Trends in the Study of Compact Objects (RETCO-II): Theory and Observation ASI Conference Series, 2015, Vol. 12, pp 14

Edited by Indranil Chattopadhyay, Anuj Nandi, Santabrata Das and Samir Mandal

\title{
Coherent Radio Emission from Pulsars
}

\author{
D. Mitra ${ }^{1 *}$, G. Melikidze ${ }^{2,3}, \&$ J. Gil ${ }^{2}$, \\ ${ }^{1}$ National Center for Radio Astrophysics, Pune, India \\ ${ }^{2}$ Kepler Institute of Astronomy, University of Zielona Gora, Lubuska 2, 65-265 Zielona Góra, Poland \\ ${ }^{3}$ Abastumani Astrophysical Observatory, Ilia State University, 3-5 Cholokashvili Ave., Tbilisi, 0160, Georgia \\ Received - ; accepted -
}

\begin{abstract}
We review a physical model where the high brightness temperature of $10^{25}-10^{30} \mathrm{~K}$ observed in pulsar radio emission is explained by coherent curvature radiation excited in the relativistic electron-positron plasma in the pulsar magnetosphere.
\end{abstract}

Keywords : radiation mechanisms: non-thermal, plasmas

\section{Introduction}

The pulsar magnetosphere needs a minimum charge density (Goldreich \& Julian 1968 ) of $n_{G J}=\Omega \cdot B / 2 \pi c$ (frequency $\Omega=2 \pi / P, \mathrm{P}$ is the pulsar period, $B$ is the magnetic field and $c$ is velocity of light) to maintain corotation. The process by which plasma is generated and flows globally in the magnetosphere is a matter of intense research (see Spitkovsky 2011), however in all models the pulsar radio emission arises due to growth of plasma instabilities in the relativistic electron positron plasma streaming along curved open dipolar magnetic field lines. Here we briefly discuss the basic observations that constrain the pulsar radio emission, followed by a proposed model for the radio emission.

\section{The radio observational Constraints and the model}

The pulsar emission consists of a bright main pulse (MP); sometimes an interpulse (IP) located $180^{\circ}$ away from the MP; occasionally pre/post-cursor (PC) emission connected via a bridge to the MP and the unpulsed emission called off-pulse emission (see left Fig 1). The MP, IP and PC emission are highly polarized. The linear

*email: dmitra@ncra.tifr.res.in 

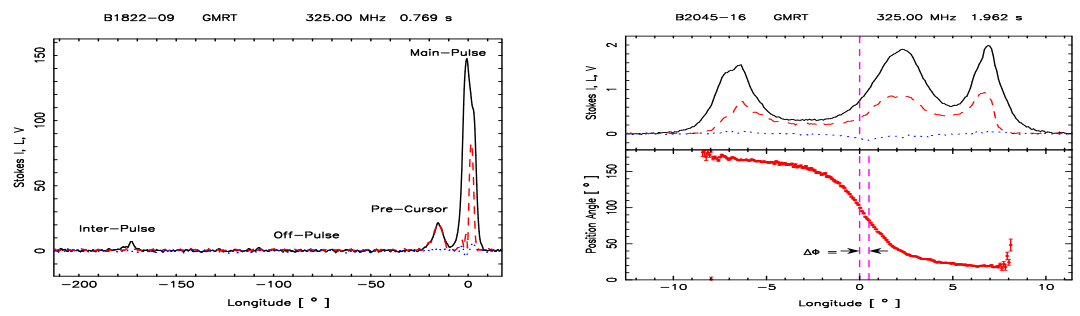

Figure 1. The left panel shows the average pulse profile of PSR B1822-09 where the emission components like MP, IP, PC are seen. The red and blue curves correspond to the linear and circular polarization. The right panel shows the MP for PSR B2045-16 (period $1.9 \mathrm{sec}$ ). Top panel: Total intensity (black), linear (red) and circular (blue). Bottom panel shows the average polarization position angle (PPA). An S-shape as predicted by the RVM is visible. The left dashed line runs through the center of the total intensity profile and the right dashed line runs through the center (inflexion point) of the PPA traverse. The range $\Delta \phi \sim 0.5^{\circ}$ is due to A/R delay which gives the radio emission height $h \sim \frac{c}{4} \frac{\Delta \phi P}{360^{\circ}} \mathrm{km}$ as about $300 \mathrm{~km}$ (see Blaskiewicz, Cordes \& Wasserman (1991); Mitra \& Li (2004)).

polarization position angle (PPA) below the MP for a large number of pulsars are in very good agreement with the rotating-vector model (RVM, Radhakrishnan \& Cooke 1969) which predicts that the PPA's are consistant with emission arising from open dipolar field lines (see Fig. 11. Here we will discuss the model that explains the MP emission of normal pulsars having periods longer than $100 \mathrm{msec}$ and dipolar magnetic fields of $\sim 10^{12}$ Gauss. Two most crucial observations that constrain the MP emission are as follows. (1) Emission heights: The radio emission originates at heights less than $10 \%$ of the light cylinder implying brightness temperatures of about $10^{25}-10^{30}$ $\mathrm{K}$. An example for measuring radio emission heights due to special relativistic effect of aberration and retardation (A/R) is explained in Fig. 1. (2) Polarization of outgoing radiation: $\mathrm{X}$-ray and radio polarization provide evidence that the emerging radiation is polarized parallel or perpendicular to the dipolar magnetic field line planes (Lai, Chernoff \& Cordes (2001))

The Model:Coherent curvature radiation has been considered as a natural emission mechanism for the observed pulsar radiation (Ruderman \& Sutherland 1975, RS75). The basic physical idea in this model is the formation of an inner vacuum gap (IVG) near the pulsar polar cap where non-stationary spark associated relativistic $\left(\gamma_{p} \sim 10^{6}\right)$ primary particles are generated. These particles further radiate in strong magnetic field and the photons thereby produce secondary $\mathrm{e}^{+} \mathrm{e}^{-}$plasma with $\gamma_{s} \sim 400$. Thus the charge density in the secondary plasma $n_{s}$ gets multiplied by a factor $\kappa=n_{s} / n_{G J} \sim 10^{4}$. Growth of plasma instabilities in the secondary plasma leads to formation of charged bunches (solitons) which can emit curvature radiation due to acceleration in curved magnetic fields. However several key issues in the RS75 model had remained unsolved for a long time. For example observations of hot xray polarcaps and subpulse drift (not discussed here) has led to the conjecture that IVG is in fact partially screend gap (PSG model by Gil et al. 2004). Further the theory of form- 


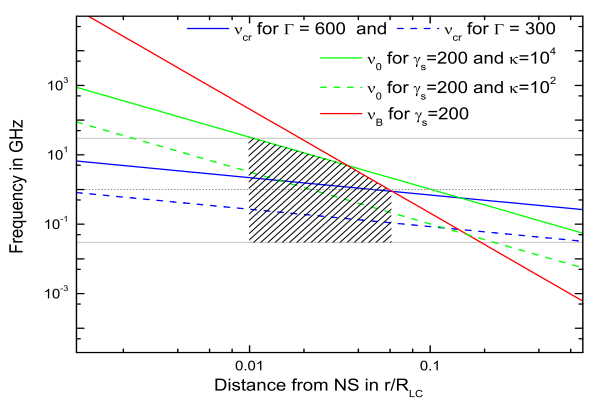

Figure 2. The figure shows the secondary plasma properties as a function of $\mathfrak{R}$ for a pulsar with $P=1 \mathrm{sec}$ and $\dot{P}=10^{-15}$. Red line: cyclotron frequency, Green line: plasma frequency, Blue line: curvature radiation. The shaded region $v_{c r}<v_{\circ}$ is where radio emission can be generated (see Paper IV for details).

ation of charged bunches leading to curvature radiation in plasma was not developed. Recently in a series of paper Melikidze, Gil \& Pataraya (2000), Gil, Luybarsky \& Melikidze (2004), Mitra, Gil \& Melikidze (2009) and Melikidze, Mitra \& Gil (2014) (Paper I-IV hereafter) we have attempted to develop a self-consistant theory of coherent curvature radiation for pulsars. Here we will only discuss the condition in the secondary plasma that leads to coherent curvature radiation.

Fig. 2 shows the plasma properties of secondary electron positron plasma as a fraction of distance $\mathfrak{R}$ to the light cylinder (i.e.: $\mathfrak{R}=h / R_{L C}$, where $R_{L C}=P c / 2 \pi$ ) for a pulsar with $P$ of 1 second and period derivative $\dot{P}$ of $10^{-15} \mathrm{~s} / \mathrm{s}$. Note that the plasma frequency, cyclotron frequency and the characteristic frequency of the soliton curvature coherent radiation can be expressed in terms of $P$ and $\dot{P}$. In the figure the plasma frequency $v_{\circ}$ (in green), cyclotron frequency $v_{B}$ (in red) and the characteristic frequency $v_{c r}$ of the soliton curvature coherent radiation (in blue) is shown (see Paper I and IV). The shaded region is the range where the pulsar radiation can be generated.

The mechanism of coherent radio emission The only plasma instability that can arise at altitudes lower than $10 \%$ of the light cylinder is the two-stream instability, as all other instabilities are suspended by the strong magnetic field. The two-stream instability is a result of the effective energy exchange between particles and waves, that can occur if the phase velocity of waves $(\omega / k)$ is near to the velocity of resonant particles $\left(v_{r}\right)$, i.e. the resonant condition $\left(\omega-k v_{r}\right)=0$ is satisfied. Such conditions can naturally be realized if a plasma is produced via non-stationary gap discharge. The spark discharge timescale in the PSG is a few tens of microseconds and this results in overlapping of successive clouds of outflowing secondary plasma. Each elementary spark-associated plasma cloud has a spread in momentum and the overlapping of particles with different momentum leads to two stream instability in the secondary plasma cloud. This triggers strong Langmuir turbulence in the plasma and if this turbulence is strong enough, the waves become modulationally unstable. The unstable wave packet described by the nonlinear Schrödinger equation leads to formation of a 
quasi-stable nonlinear solitary wave, i.e. a soliton (Paper I). The longitudinal (along k) size of the soliton should be much larger than the wavelength of the linear Langmuir wave. Also it has to be charged to be able to radiate coherent curvature emission. Thus, the soliton bunch has to be charge separated, which can be caused either by difference in the distribution function of electrons and positrons, or by admixture of iron ions in the secondary plasma or by both these effects. A sufficient number of charged solitons is formed which can account for the observed radio luminosity in pulsars (Paper I). The wavelength of the emitted waves should be longer than the longitudinal size of the soliton $\Delta$. This is the necessary condition for the coherency of a curvature radiation process. Thus, the frequencies plotted in Fig. 2 should obey the following constraints $v_{\mathrm{cr}}<\frac{c}{\Delta} \ll v_{\circ} \ll v_{B}$. It is clearly seen from Fig. 2 that the observed pulsar radiation cannot be generated at altitudes exceeding $10 \%$ of the light cylinder radius (practically the radio emission region should be contained between one to several percent of $R_{L C}$; see dashed area in Fig. 2). This conclusion is based purely on the properties of plasma and the emission mechanism. And it corresponds perfectly to the other limits on emission heights obtained from observations. (e.g. Blaskiewicz, Cordes \& Wasserman 1991; Mitra \& Li 2004).

The curvature radiation excites the extraordinary X-mode (polarized $\perp$ to the magnetic field plane) and the ordinary O-mode (polarized $\|$ to the magnetic field plane) in the secondary plasma, and recently it was shown in Paper IV that the radiation can emerge retaining its polarization without getting affected due to propagation effects in the plasma (Paper IV). This X and O mode should hence be associated with the parallel and perpendicularly polarized emission observed in pulsars.

\section{Acknowledgements}

DM thanks the RETCO organizers for invitation to give a talk in the meeting.

\section{References}

Blaskiewicz, M., Cordes, J. M., \& Wasserman, I., 1991, ApJ, 370, 643

Gil, J., Melikidze, G. I. \& Geppert, U., 2003, A\&A, 407, 315

Gil, J., Lyubarsky, Y., \& Melikidze, G. I., 2004, ApJ, 600,872 (Paper II)

Goldreich, P \& Julian W. H., 1968, ApJ, 157, 869

Lai, D., Chernoff, D. F., \& Cordes, J. M., 2001, ApJ, 549, 1111

Melikidze, G. I, Gil, J., \& Pataraya, A. D. 2000, ApJ, 544, 1081 (Paper I)

Melikidze, G. I., Mitra, D. \& Gil, J., 2014, 794, 105 (Paper IV)

Mitra, D., Gil, J. \& Melikidze, G. I., 2009, ApJ, 696L, 141 (Paper III)

Mitra, D. \& Li, X. H., 2004, A\&A, 421, 215

Radhakrishnan V., \& Cooke D. J., 1969, ApJL, 3, 225

Ruderman, M. A., \& Sutherland, P. G., 1975, ApJ, 196, 51

Spitkovsky, A, 2011, HEEP, editor Torres, D. F \& Rea, N., 139 